\title{
Correlation of syntaxin-1 and SNAP-25 clusters with docking and fusion of insulin granules analysed by total internal reflection fluorescence microscopy
}

\author{
M. Ohara-Imaizumi ${ }^{1}$ C. Nishiwaki ${ }^{1}$ Y. Nakamichi ${ }^{1}$-T. Kikuta ${ }^{1}$ S. Nagai ${ }^{1}$ S. Nagamatsu ${ }^{1}$ \\ ${ }^{1}$ Department of Biochemistry, Kyorin University School of Medicine, Tokyo, Japan
}

\begin{abstract}
Aims/hypothesis. The interaction of syntaxin-1 and SNAP-25 with insulin exocytosis was examined using the diabetic Goto-Kakizaki (GK) rat and a total internal reflection fluorescence (TIRF) imaging system.

Methods. Primary rat pancreatic beta cells were immunostained with anti-syntaxin-1A, anti-SNAP-25 and anti-insulin antibodies, and then observed by TIRF microscopy. The real-time image of GFP-labelled insulin granules motion was monitored by TIRF.

Results. The number of syntaxin-1A and SNAP-25 clusters, and the number of docked insulin granules on the plasma membrane were reduced in GK beta cells. When GK rats were treated with daily insulin injection for 2 weeks, the number of syntaxin-1 and SNAP-25
\end{abstract}

clusters was restored, along with the number of docked insulin granules. The infection of GK beta cells with Adex1CA SNAP-25 increased the number of docked insulin granules. TIRF imaging analysis demonstrated that the decreased number of fusion events from previously docked insulin granules in GK beta cells was restored when the number of docked insulin granules increased by insulin treatment or Adex1CA SNAP-25 infection.

Conclusions/interpretation. There was a close correlation between the number of syntaxin-1 and SNAP-25 clusters and the number of docked insulin granules, which is associated with the fusion of insulin granules.

Keywords Diabetes mellitus - Evanescent . Exocytosis · Fusion - Insulin release $\cdot$ SNARE $\cdot$ TIRF

\section{Introduction}

The fundamental components of the secretory machinery required for the docking and fusion of vesicles with the plasma membrane have been revealed $[1,2]$.

Received: 10 May 2004 / Accepted: 5 August 2004

Published online: 11 December 2004

(C) Springer-Verlag 2004

S. Nagamatsu ( $)$

Department of Biochemistry,

Kyorin University School of Medicine, Mitaka,

Tokyo 181-8611, Japan

E-mail: shinya@kyorin-u.ac.jp

Tel.: +81-422-475511 ext 3437, Fax: +81-422-475538

Abbreviations: GFP, green fluorescent protein -

GK, Goto-Kakizaki - NSF, $N$-ethylmaleimide-sensitive factor ·

SNAP, soluble NSF attachment protein $\cdot \mathrm{t}$-SNARE, target

membrane SNAP receptor - TIRF, total internal reflection

fluorescence
These components, including $N$-ethylmaleimide-sensitive factor (NSF), soluble NSF attachment protein (SNAP), and membrane-associated SNAP receptors (SNAREs) such as syntaxin-1 and SNAP-25, are also expressed in pancreatic beta cells $[3,4,5]$, which play an important role in insulin exocytosis $[6,7,8,9]$. We [10] and others [11, 12] have demonstrated that the expression of SNARE proteins is decreased in diabetic animal models. It is conceivable that the expression of SNARE proteins is closely related to the docking/ fusion of insulin exocytosis; however, there has been no apparent evidence to show the direct interaction between target membrane (t-)SNAREs and docking/ fusion of insulin granules.

Imaging techniques are powerful tools for detecting vesicle trafficking and spatial distribution of membrane proteins in live cells and they have provided significant advances in understanding the mechanism of exocytosis $[13,14,15]$. In particular, the use of to- 
tal internal reflection fluorescence (TIRF) microscopy (also called evanescent wave microscopy), which allows fluorescence excitation within a closely restricted domain close to the plasma membrane (within $100 \mathrm{~nm}$ ) [16], has permitted us to observe not only single granule movement underlying exocytosis $[17$, 18], but also the single molecules on the plasma membrane $[19,20]$. Thus, we were able to observe using TIRF microscopy with high resolution, the single insulin granules approaching, docking, and fusing with the plasma membrane, and the spatial localisation of t-SNAREs such as syntaxin-1 and SNAP-25 in the plasma membrane of live cells.

In the present study, a TIRF system was utilised to address the question of whether t-SNAREs are related to the docking and fusion of insulin granules using insulin-treated and/or untreated diabetic Goto-Kakizaki (GK) beta cells where the expression levels of t-SNAREs and docking/fusion of insulin granules were changed. We demonstrate that t-SNAREs are closely associated with the number of docked insulin granules, in parallel with the fusion events from previously docked granules.

\section{Materials and methods}

Cells. Diabetic GK rats and non-diabetic male Wistar rats were obtained from a commercial breeder (Oriental Yeast, Tokyo, Japan). The rats were given free access to food and water until the start of experiments, which were conducted with 10-weekold male rats. The body weight of GK rats was not statistically different from that of controls. The plasma glucose concentration in the fed state, measured by the glucose oxidase method, was $12.3 \pm 0.78 \mathrm{mmol} / \mathrm{l}(n=16)$ in $\mathrm{GK}$ rats and $5.8 \pm 0.61 \mathrm{mmol} / \mathrm{l}$ $(n=18)$ in control rats respectively $(p<0.0001)$. For the normalisation of hyperglycaemia, human insulin (Humalin N; Lilly, Indianapolis, Ala., USA) was injected subcutaneously at 08.00 ( $2 \mathrm{U} / \mathrm{rat}$ ) and 20.00 (4 U/rat) hours daily for 2 weeks. Pancreatic islets of Langerhans were isolated by collagenase digestion [10], with some modification. Isolated islets were dissociated into single cells by incubation in $\mathrm{Ca}^{2+}$-free $\mathrm{KRB}$ containing $1 \mathrm{mmol} / \mathrm{l}$ EGTA, and cultured on fibronectin-coated (KOKEN, Tokyo, Japan) high-refractive-index glass (Olympus, Tokyo, Japan) in RPMI 1640 medium supplemented with 10\% FBS (Invitrogen Gibco BRL, Carlsbad, Calif., USA), 200 U/ml penicillin, and $200 \mu \mathrm{g} / \mathrm{ml}$ streptomycin at $37{ }^{\circ} \mathrm{C}$ in an atmosphere of $5 \% \mathrm{CO}_{2}$.

Immunohistochemical analysis. Pancreatic beta cells were fixed, made permeable with $2 \%$ paraformaldehyde $/ 0.1 \%$ Triton $\mathrm{X}-100$, and processed for immunocytochemistry as described previously [9]. Cells were labelled with monoclonal anti-insulin antibodies (Sigma-Aldrich, St. Louis, Mo., USA), antiHPC1-antibodies (Sigma-Aldrich), and anti-SNAP-25 antibodies (Wako, Osaka, Japan), and then processed with Cy3-conjugated anti-mouse IgG (Amersham Biosciences, Little Chalfont, UK). F-actin was stained by incubation with fluorescein isothiocyanate-conjugated phalloidin (Sigma-Aldrich). For double-immunostaining study, syntaxin-1 was stained with polyclonal anti-HPC-l-antiserum [5] and fluorescein anti-rabbit IgG (Jackson Immuno Research Laboratories, Bar Harbor,
Me., USA). Immunofluorescence staining was detected by TIRF or epifluorescence microscopy.

TIRF microscopy. The Olympus total internal reflection system was used with minor modifications as described previously [18]. Light from an Ar laser (488 nm) or an He/Ne laser $(543 \mathrm{~nm})$ was introduced to an inverted epifluorescence microscope (IX70, Olympus) through a single-mode fibre and two illumination lenses; the light was focused at the back focal plane of a high-aperture objective lens (Apo 100× OHR; NA 1.65 , Olympus). To observe the fluorescence image of $\mathrm{Cy} 3$, we

\section{a}
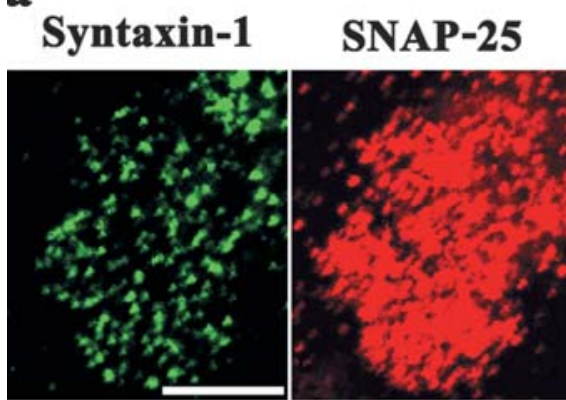

Overlay

Insulin granule

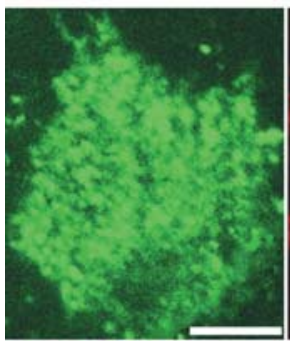

SNAP-25
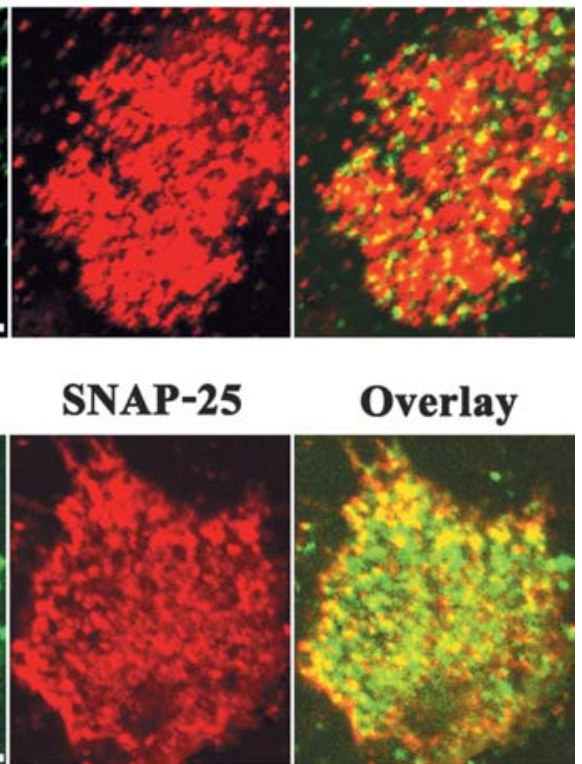

Overlay

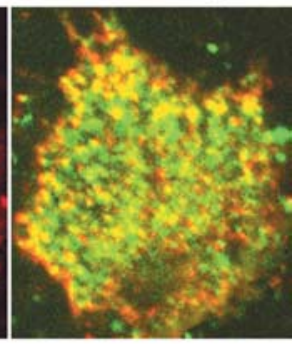

b
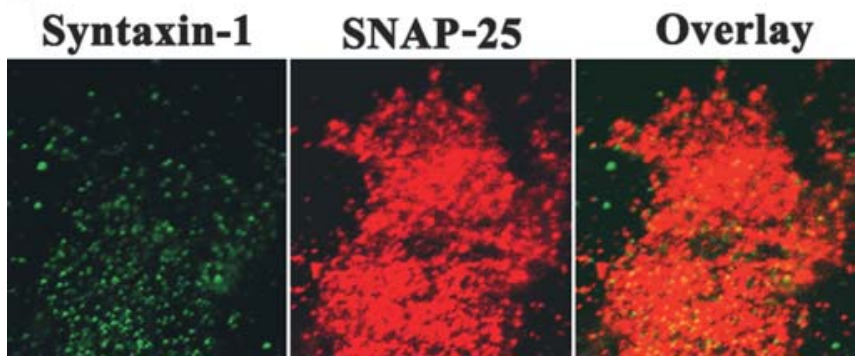

Fig. 1. SNAP-25 colocalised with syntaxin-1 and insulin granules on the plasma membrane of a primary rat pancreatic beta cell. a. TIRF images in a normal primary beta cell. After normal rat pancreatic beta cells were prepared, they were fixed, immunostained for syntaxin-1, SNAP-25 or insulin, and viewed simultaneously by TIRF microscopy as described [23]. The colocalisation of syntaxin-1 and SNAP-25, and of SNAP25 and insulin granules is demonstrated by the superposition (yellow colour) of red and green channel images. b. TIRF image of SNAP-25-infected diabetic GK beta cell plasma membrane. Two days after GK beta cells were infected with Adex1CA SNAP-25, they were fixed, immunostained for syntaxin-1 and SNAP-25, and viewed by TIRF microscopy. The overlay of the images shows that most of the syntaxin-1 clusters were overlapped on SNAP-25 clusters 
used a 543-nm laser line and a long-pass 590-nm filter. To observe green fluorescent protein (GFP) or fluorescein, we used a 488-nm laser line for excitation and a $515-\mathrm{nm}$ pass filter for the barrier. The procedure for monitoring the GFP-labelled insulin granule motion in primary rat pancreatic beta cells is described elsewhere [21]. Briefly, primary beta cells expressing insulin-GFP on the glass cover slip (Olympus) were mounted in an open chamber and then transferred to the thermostat-con-

a
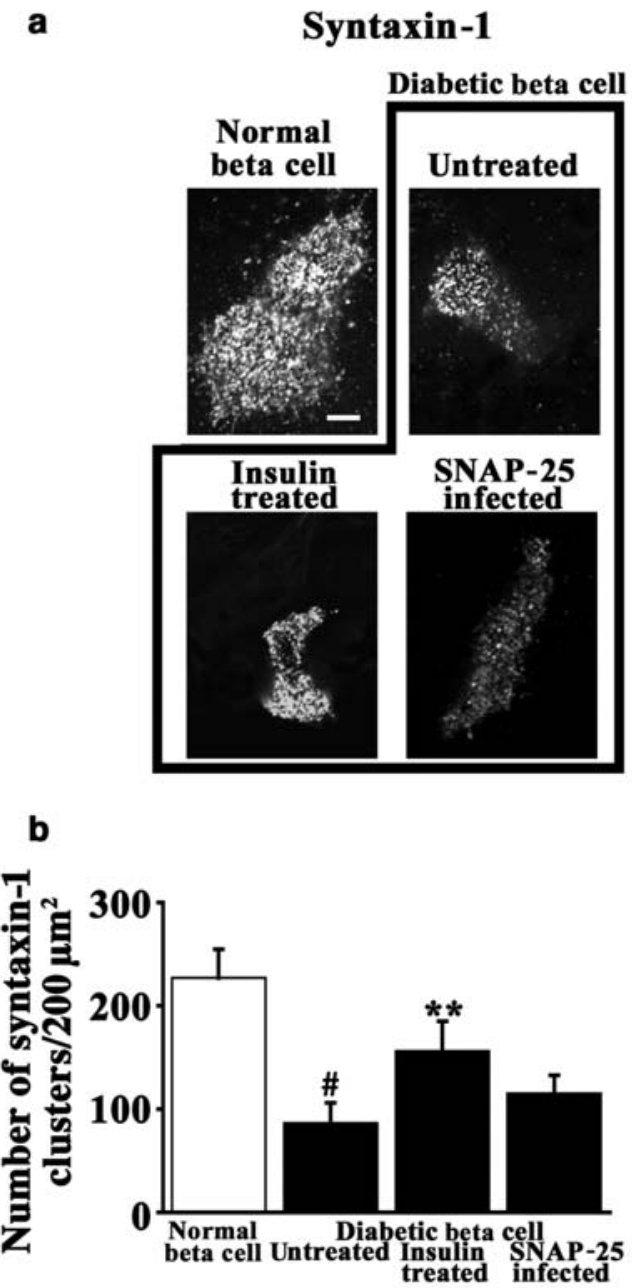

C

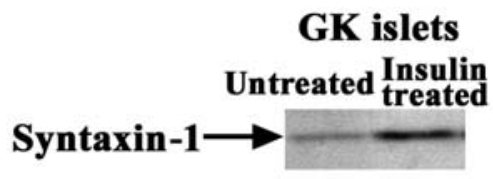

Fig. 2. TIRF images, and the immunoblot analysis of syntaxin1 and SNAP-25 in normal and diabetic GK beta cells. a. Effects of insulin treatment and Adex1CA SNAP-25 infection on syntaxin-1 and SNAP-25 on the plasma membrane of diabetic GK beta cells were shown by TIRF images. Insulin-treated group: GK rats were treated with and/or without a daily subcutaneous injection of human insulin for 2 weeks, and then pancreatic beta cells were prepared. Adex1CA SNAP-25 infected group: the pancreatic beta cells prepared from GK rats were infected with Adex1CA SNAP-25 or Adex 1w (empty virus) and cultured for 2 days as described in Materials and methods. Treated GK or non-treated normal pancreatic beta cells were prepared and fixed, and then immunostained for syntaxin-1 and SNAP-25 before being observed by TIRF microscopy. The trolled stage $\left(37^{\circ} \mathrm{C}\right)$. Cells were stimulated by $22 \mathrm{mmol} / \mathrm{l}$ glucose (final), and the measured penetration depths were about $45 \mathrm{~nm}$.

Preparation of recombinant adenoviruses and adenovirusmediated gene transduction. The construction of expression vectors and recombinant adenovirus encoding insulin-GFP and SNAP-25 has been previously described [10, 21]. To label
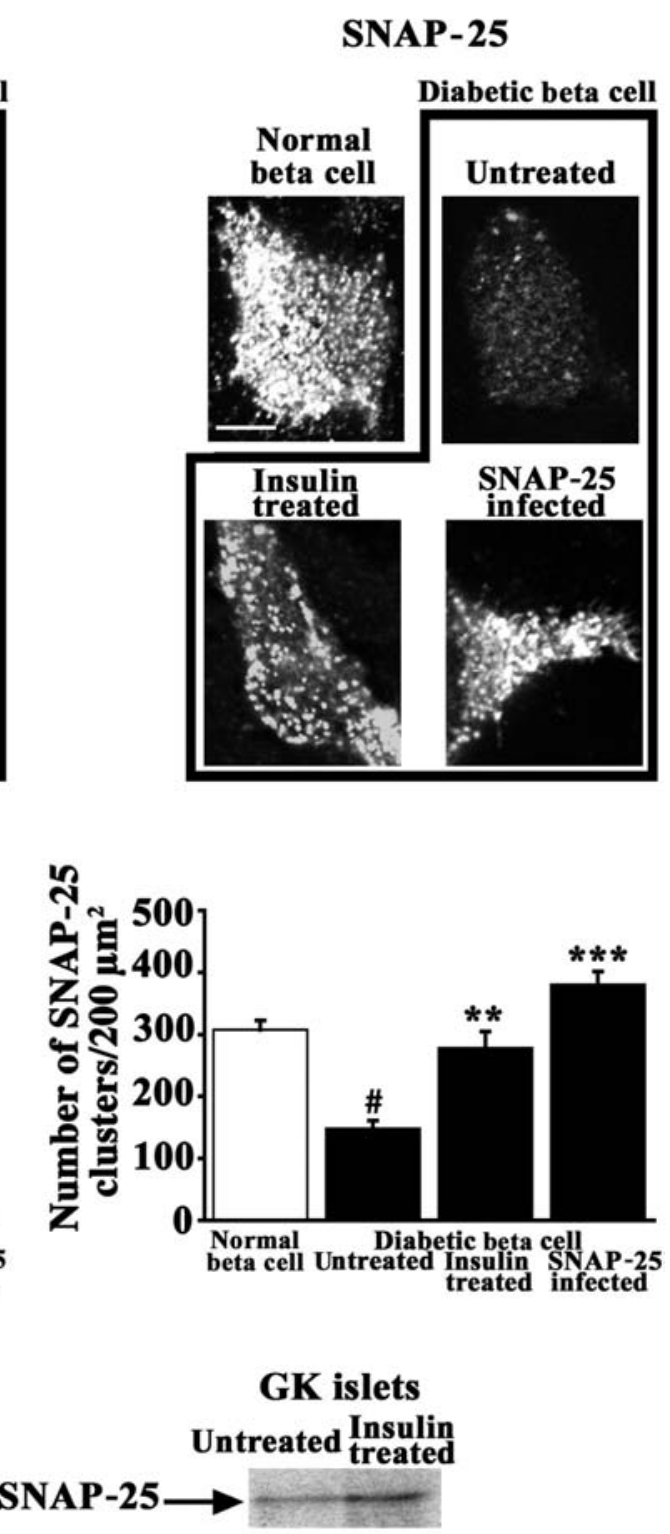

scale bars represent $5 \mu \mathrm{m}$. Each image is representative of separate experiments. b. The number of syntaxin-1 and SNAP-25 clusters in the plasma membrane. Individual fluorescent spots of syntaxin-1 and SNAP-25 shown in TIRF images were counted (normal beta cells, $n=10$; diabetic beta cells, $n=8$ ). The number of clusters was calculated per $200 \mu \mathrm{m}^{2}$. \# $p<0.01$ vs normal, $* * p<0.01$ vs untreated, $* * * p<0.001$ vs untreated beta cells. c. Immunoblot analysis. Islet proteins (200 islets) were extracted from insulin-treated and -untreated GK rat pancreas, subjected to SDS-PAGE, and immunoblotted with the indicated antibodies, which was followed by chemiluminescence reaction. Each immunoblot is a representation of three separate experiments. The levels of the protein bands were determined using the NIH Image program 
the insulin secretory granules, cultured single cells were incubated with RPMI 1640 medium (5\% FBS) and the required adenovirus (Adex1CA insulin-GFP, 30 MOI per cell) for $1 \mathrm{~h}$ at $37{ }^{\circ} \mathrm{C}$, after which RPMI 1640 medium with $10 \%$ FBS was added. For SNAP-25 infection study, cultured single cells were infected with Adex1CA SNAP-25 (20 MOI per cell) prior to labelling the granules with Adex1CA insulin-GFP. Experiments were performed 2 days after the final infection.

Acquiring the images and analysis. Images were collected by a cooled charge-coupled-device camera (Micromax, MMX-512BFT; Roper Scientific, Princeton Instruments, Trenton, N.J., USA) operated with Metamorph 4.6; Universal Imaging, Downingtown, Pa., USA) as described previously [21]. Most analyses, including counting the number of fluorescent spots, tracking (the single projection of different images) area calculations and fluorescent intensity, were performed using Metamorph software. To analyse the fusion data, fusion events were manually selected, and the average fluorescence intensity of individual granules in a $1-\mu \mathrm{m} \times 1-\mu \mathrm{m}$ square placed over the granule centre was calculated. The number of fusion events was manually counted while looping 15,000 frame time-lapses. TIRF images were finally exported as single TIFF files and were further processed using Adobe Photoshop 6.0.

Immunoblotting. Islets prepared from insulin-treated and/or insulin-untreated GK or normal Wistar rats were disrupted by sonication, boiled in SDS sample buffer with $10 \mathrm{mmol} / 1$ dithiothreitol, subjected to SDS-PAGE, and then transferred onto nitrocellulose filters. Immunoblotting procedures were performed as described previously [10] using anti-HPC-1-antibodies, anti-SNAP-25 antibodies, and anti-actin monoclonal antibody (Chemicon International, Temecula, Calif., USA). The protein bands were scanned and analysed by NIH Image.

Insulin release from islets. GK islets prepared before and after 2 weeks of daily insulin injection were preincubated with $2.2 \mathrm{mmol} / 1$ glucose in KRB for $1 \mathrm{~h}$. They were then challenged with $22 \mathrm{mmol} / \mathrm{l}$ glucose plus forskolin $(20 \mu \mathrm{mol} / \mathrm{l})$. The media were collected at the end of the challenge period, then analysed for immunoreactive insulin by radioimmunoassay as previously described [9].

Statistical analysis. Results are means \pm SEM, and statistical analysis was performed using ANOVA followed by Fisher's test and regression analysis using the Statview software (Abacus Concepts, Berkeley, Calif., USA).

\section{Results}

Decrease in the number of t-SNARE clusters and docked insulin granules in diabetic GK beta cell plasma membrane. A recent study using PC12 cells reported that t-SNAREs are concentrated in separate clusters, shown using membrane-sheet procedures [22]. Consistent with their results, we previously observed, using TIRF microscopy, that t-SNAREs are distributed in numerous spots in the MIN6 cell plasma membrane [23]. In the present study we examined this issue using rat primary pancreatic beta cells. As shown in Fig. 1a, the immunofluorescence of syntaxin-1 and SNAP-25 on the plasma membrane of rat primary pancreatic beta cells was distributed in numerous spots as well as in those observed in MIN6 cells.
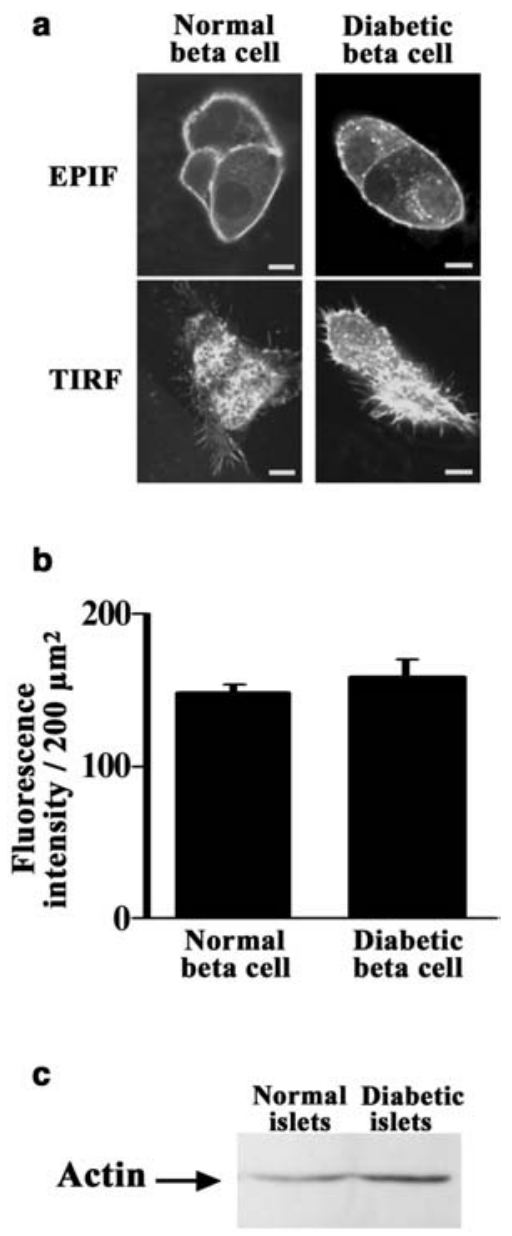

Fig. 3. Immunohistochemistry and immunoblot of actin in normal and diabetic GK beta cells. a. TIRF and epifluorescence (EPIF) images of F-actin on the beta cell plasma membrane. After pancreatic beta cells were prepared from normal and GK rat pancreas, F-actin was stained with phalloidin-fluorescein isothiocyanate and observed by TIRF and EPIF microscopy. Each image is representative of three separate experiments. b. The fluorescence intensity of phalloidin-fluorescein isothiocyanate analysed by Metamorph based on TIRF image $(n=5$ cells). c. Immunoblot analysis. Islet proteins were subjected to SDS-PAGE, immunoblotted with anti-actin monoclonal antibody, and scanned as described in the legend of Fig. 2 ( $n=3$ for separate experiments)

The apparent diameters of the syntaxin-1 and SNAP-25 clusters were approximately $400 \mathrm{~nm}$, which is similar to that reported in MIN6 cells [23]. Dual-stained immunohistochemical studies for syntaxin-1 and SNAP-25 showed that most of the syntaxin-1 clusters were colocalised with the SNAP-25 clusters, which were also colocalised with insulin granules docked on the plasma membrane (Fig. 1a). Thus, the characterisation of t-SNAREs on the plasma membrane appears not to be different between insulinoma MIN6 cells and primary pancreatic beta cells. We then examined the change of t-SNARE clusters in diabetic GK beta cells. In diabetic GK beta cells, TIRF images and their analyses showed that the number of clusters of syntaxin-1A and SNAP-25 decreased to less than half of that in 

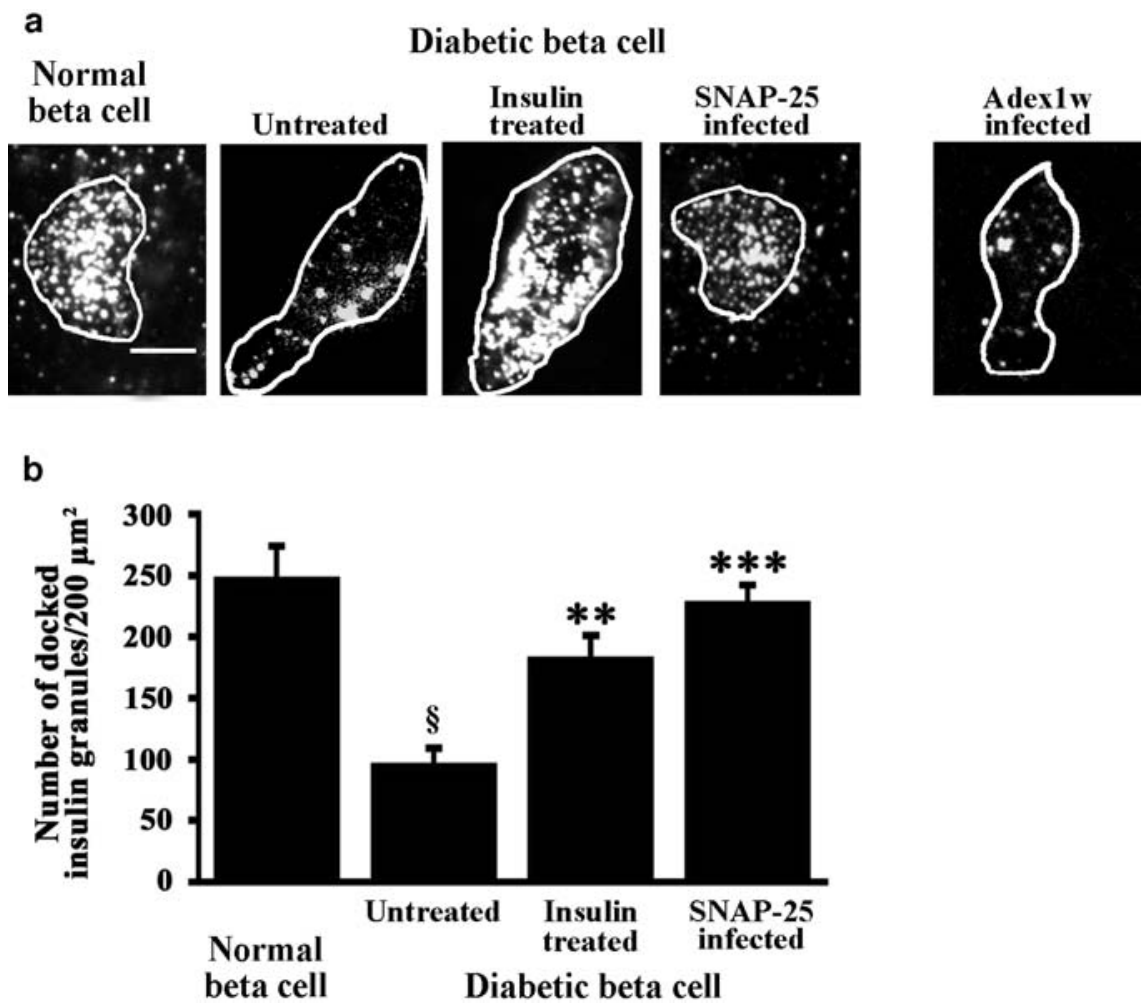

Fig. 4. Histochemical study of insulin secretory granules docked to the plasma membrane. a. TIRF images of docked insulin granules in normal and diabetic GK beta cells. For insulin treatment and Adex1CA SNAP-25 or Adex1w infection, GK rats and beta cells were processed as described in the legend of Fig. 2. Cells were fixed with paraformaldehyde, then immunostained for insulin. The surrounding lines represent the outline of cells that are attached to the cover glass. Representative images are shown. The scale bar represents $5 \mu \mathrm{m}$. Note that infection with Adex $1 \mathrm{w}$ empty virus did not affect the docked insulin granules. b. The number of insulin granules morphologically docked to the plasma membrane. Individual fluorescent spots shown as TIRF images were counted and calculated per $200 \mu \mathrm{m}^{2}$ (normal beta cells, $n=15$; GK beta cells, $n=8)$. $\S p<0.0001$ vs normal beta cells, $* * p<0.01$ vs untreated diabetic beta cells, $* * * p<0.001$ vs untreated diabetic beta cells

normal beta cells (Fig. 2a, b). To examine whether the decreased number of t-SNARE clusters in diabetic beta cells is a specific phenomenon, we immunostained F-actin as a control. As shown in Fig. 3, no differences in ring-shaped F-actin immunostaining or actin immunoblotting were observed between normal and diabetic beta cells, indicating that the alteration in the number of t-SNARE clusters in the diabetic GK beta cell is a specific phenomenon. Although actin signals in GK islets detected by immunoblot had a tendency to increase, no statistically significant difference was found between normal and GK diabetic islets by scanning and analysis of protein bands with NIH Image ( $100 \%$ vs $148 \pm 39 \%$; $n=3$ ).

We then examined the change in insulin granules morphologically docked to the plasma membrane in diabetic GK beta cells. There is no precise definition of "morphologically docked granules", but the following are generally used: (i) vesicle is located within $200 \mathrm{~nm}$ of the plasma membrane, shown by electron microscopy [24]; (ii) vesicle is located within $100 \mathrm{~nm}$ of the surface membrane, shown by electron microscopy [25]; (iii) vesicle distance from plasma membrane is less than $10 \mathrm{~nm}$, shown by TIRF microscopy [26]; and (iv) vesicle is in direct contact with the plasma membrane, shown by electron microscopy [27]. As the penetration depth under our TIRF condition is $80 \mathrm{~nm}$, we defined "morphologically docked granules" as the vesicle being located within $100 \mathrm{~nm}$ of the plasma membrane. As shown in Fig. 4a, TIRF imaging depicted the single insulin granules morphologically docked to the plasma membrane, whereby we could manually count the number of docked insulin granules in normal and diabetic beta cells. As shown in Fig. 4b, the number of insulin granules docked to the plasma membrane was markedly reduced in diabetic GK beta cells.

Recovery of the number of t-SNARE clusters and docked insulin granules by insulin treatment. We addressed the question of whether the normalisation of blood glucose levels affects the number of t-SNARE clusters and the number of docked insulin granules in GK beta cells. We treated GK rats with daily insulin injection for 2 weeks, resulting in the reduced blood glucose levels (untreated 11.2 \pm 0.61 vs treated $5.6 \pm 0.33 \mathrm{mmol} / \mathrm{l})$. After 2 weeks of insulin treatment, pancreatic beta cells were prepared and immunostained with anti-syntaxin-1A, anti-SNAP-25, and anti-insulin antibodies. The number of syntaxin-1 and 
SNAP-25 clusters in the plasma membrane was partially, but significantly, recovered to subnormal levels (Fig. 2a, b). Immunoblot analysis also showed that the levels of syntaxin-1A and SNAP-25 were increased in islets isolated from insulin-treated GK rats (Fig. 2c; $182 \pm 24 \%$ in syntaxin- $1,165 \pm 32 \%$ in SNAP-25 when expressed as $100 \%$ in insulin-untreated GK islets, $n=3$ for each, $p<0.01)$. It is of note that along with an increase in the number of t-SNARE clusters, there was also an increase of about two-fold in the number of insulin granules docked to the plasma membrane (Fig. 4a, b).

Correlation between the fusion events and the number of docked insulin granules. We previously reported that the fusion events during first-phase release originate from previously docked granules (resident) both in MIN6 cells and primary pancreatic beta cells [18, 21]. In the present study, we tested whether the number of fusion events is correlated with the number of docked insulin granules. After pancreatic beta cells were prepared from normal rats, insulin-untreated and insulin-treated GK rats, they were infected with Adex1CA insulin-GFP to label the insulin secretory granules. After 2 days of infection, granule motion was monitored using TIRF by $22 \mathrm{mmol} / \mathrm{l}$ glucose stimulation. In insulin-untreated diabetic GK beta cells, the fusion events from previously docked granules were rarely observed (Fig. 5; untreated). In contrast, in insulin-treated GK beta cells, high glucose stimulation caused fusion events from previously docked insulin granules (Fig. 5; treated) where the number of docked insulin granules was recovered to subnormal levels (see Fig. 4). Furthermore, we measured insulin release from GK islets before and after insulin treatment. Consistent with the results of the TIRF imaging analysis, glucose-stimulated insulin release from insulin-treated GK islets was slightly improved compared with before treatment $\left(1.5 \pm 0.3 \mathrm{ng} \cdot\right.$ islet $^{-1} \cdot \mathrm{h}^{-1}$ before treatment vs $2.1 \pm 0.4 \mathrm{ng}$ islet $^{-1} \cdot \mathrm{h}^{-1}$ after treatment, $p<0.01, n=3$ for separate experiments).

Rescue of fusion events by recovering the number of docked insulin granules and restoring SNAP-25 clusters to normal levels. In order to examine whether the concentration of t-SNARE clusters is linked to the docking status followed by subsequent fusion of insulin granules, we restored the decreased number of SNAP-25 clusters to normal levels by infecting GK beta cells with Adex1CA SNAP-25. GK beta cells infected with Adex1CA SNAP-25 showed the normalised number of SNAP-25 clusters on the plasma membrane (Fig. 2a, b) where the decreased number of docked insulin granules was also restored to subnormal levels (Fig. 4). However, infection with Adex1CA SNAP-25 did not affect the number of syntaxin-1 clusters (Fig. 2a). GK beta cells infected with empty
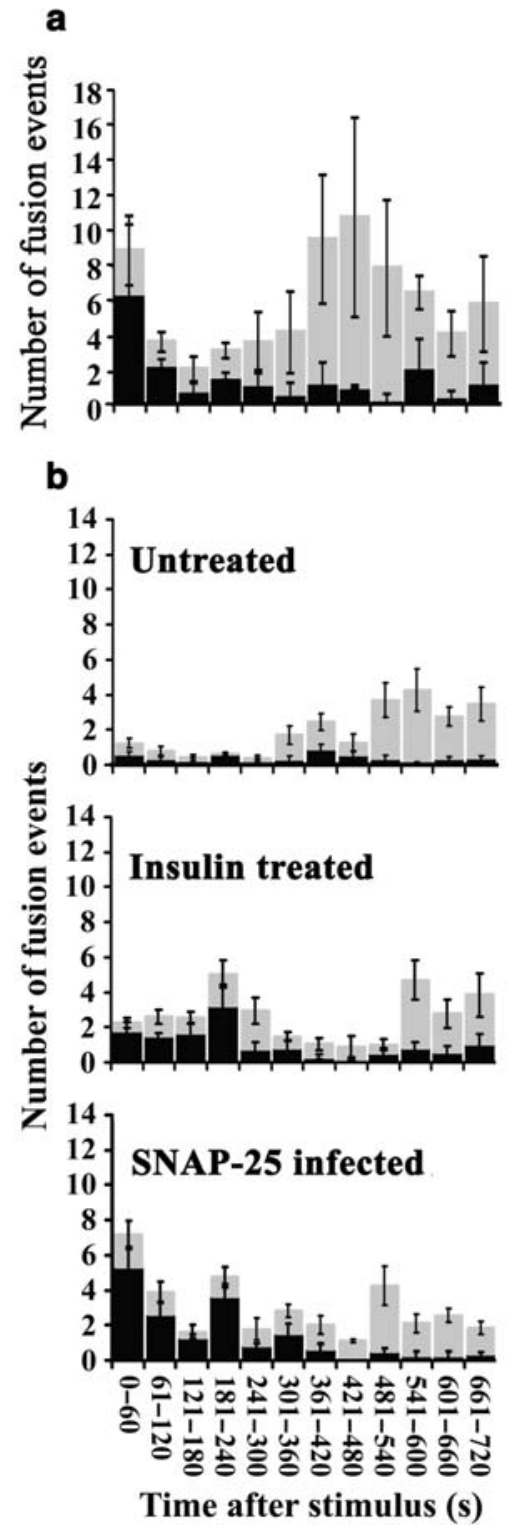

Fig. 5. Restoration of the decreased number of fusion events in normal (a) and diabetic (b) beta cells. Black bars, resident; grey bars, newcomer. For insulin treatment and SNAP-25 infection, GK beta cells were prepared as in the legend of Fig. 2. After these procedures, beta cells were infected with Adex 1CA insulin-GFP in order to label the insulin granules, then the real-time motion of GFP-labelled insulin granules during stimulation with $22 \mathrm{mmol} / \mathrm{l}$ glucose was imaged close to the plasma membrane by TIRF, and fusion was analysed by Metamorph software. The graph shows the histogram of the number of fusion events (per $200 \mu \mathrm{m}^{2}$; normal beta cells, $n=5$; GK beta cells, $n=4$ )

virus Adex 1w did not show any change in the number of SNAP-25 clusters (data not shown) or in the number of docked insulin granules (Fig. 3a). As shown in Fig. 1b, most of the syntaxin-1 clusters were colocalised with SNAP-25 clusters restored by Adex1CA SNAP-25 infection in GK beta cells. Cotransfection of Adex1CA SNAP-25 with Adex1CA syntaxin-1A did not affect the subnormalised number of docked insulin 
granules (data not shown). We then performed TIRF imaging analysis of the docking and fusion of insulin granules stimulated by $22 \mathrm{mmol} / \mathrm{l}$ glucose using Adex1CA-SNAP-25-infected GK beta cells. These beta cells showed a marked increase in fusion events from previously docked granules (Fig. 5; SNAP-25 infected).

\section{Discussion}

In the present study, we examined the interaction between the number of t-SNAREs and the number of insulin granules docked to the plasma membrane, and between the number of docked insulin granules and the number of fusion events. Our data demonstrated a close correlation of t-SNARE clusters with docked insulin granules, and that the number of docked insulin granules was correlated with the fusion events from previously docked granules.

TIRF imaging showed that the number of syntaxin1A and SNAP-25 clusters and the number of docked insulin granules on the plasma membrane decreased in GK beta cells. It is of note that the recovery of the decreased number of SNAP-25 clusters in GK beta cells to normal levels by adenovirus treatment or insulin treatment could restore the number of docked insulin granules, which caused an increased number of fusion events from previously docked granules. We previously reported that restoration of decreased t-SNARE levels improved the impaired insulin secretion in GK islets [10]. At that time, however, we could not know how t-SNARE restoration affects the insulin exocytosis. Now, we know that the restored number of SNAP25 clusters subnormalised the number of docked insulin granules, through which the fusion from previously docked granules was probably recovered. Thus, in diabetic GK beta cells, the decreased number of t-SNARE clusters may result in impaired insulin granule docking status followed by a decreased fusion event that might lead to the loss of first-phase insulin release. We have recently reported that the disruption of t-SNARE clusters by cholesterol depletion with methyl- $\beta$-cyclodextrin treatment causes the inhibition of docking and fusion of insulin granules [23] and thus indicates that impaired formation of t-SNARE clusters in diabetic GK beta cells may be involved in the decreased docking and fusion events of insulin granules.

It is interesting that the transduction with Adex $1 \mathrm{CA}$ SNAP-25 alone was sufficient to recover the reduced number of docked insulin granules in diabetic GK beta cells, which was followed by an increase in fusion events. Indeed, we had found that the transduction of GK islets with Adex1CA SNAP-25 alone recovered the glucose-stimulated insulin release effectively [10]. Although the precise reason why the restoration of SNAP-25 clusters alone recovered the im- paired docking and fusion events in spite of the imbalance in relative levels of SNAP-25 and syntaxin-1 is not known at present, SNAP-25 may be a strict requirement more so than syntaxin-1 in evoked fusion event, as recently shown [28, 29]. Further studies about this important issue are required.

The present study also reported the interesting finding that insulin treatment improved the impaired docking and fusion events in GK beta cells. Although one clinical study showed that the impaired insulin release in diabetic patients was improved when the hyperglycaemia was normalised by daily insulin injection [30], there have been no in vitro studies to examine its mechanism in detail. Judging from our data, it is conceivable that insulin treatment may have reduced the insulin secretion and thus allowed beta cells to increase the number of docked granules. However, because the effects of insulin treatment persisted for at least 2 days during culture period after beta cell preparation, these would be trophic effects. Another simple explanation would be that improved glucotoxicity by insulin treatment may have recovered the beta cell functions.

Finally, we calculated the total number of docked insulin granules in rat pancreatic beta cells. On the basis of our data, we estimated that normal primary beta cells contain approximately 1200 insulin granules docked to the plasma membrane. As the surface area of a beta cell is reported to be $973 \mu \mathrm{m}^{2}$ [31] and the number of docked granules of normal beta cells is calculated to be 246 granules/200 $\mu^{2}$, the total number of docked insulin granules is about 1200 . In a study that used mouse pancreas and electron microscopy [31], Dean reported that the pancreatic beta cells contain about 13,000 insulin granules, and recently, mouse and rat beta cells were reported to have about 10,000 insulin granules per cell, with an estimated number of docked granules of about 600 and 450 respectively $[32,33]$. Thus, the number of docked insulin granules is slightly larger in our TIRF imaging analysis. The discrepancy may be because (i) electron microscopy only provides a snapshot of the situation in the beta cells at the time of fixation, so it is not possible to conclude that a certain number of docked granules are really physically attached to the plasma membrane; or (ii) TIRF imaging only gives information on a small part of the cell attached to the coverglass where vesicles are located within $100 \mathrm{~nm}$ of the plasma membrane, so the calculated total number of granules docked to the whole cell surface may be an overestimation.

In conclusion, there was an interaction between the number of t-SNARE clusters and the number of docked insulin granules, which were associated with fusion events from previously docked insulin granules.

Acknowledgements. We thank I. Saito for kindly providing the adenovirus cosmid vector and parental virus. This work was supported by Grants-in-Aid for Scientific Research (C) 
14570130 (to M. Ohara-Imaizumi) and (B) 15390108 (to S. Nagamatsu) and Scientific Research on Priority Areas 16044240 (to M. Ohara-Imaizumi) from the Japanese Ministry of Education, Culture, Sports, Science and Technology, and by a grant from the Japan Private School Promotion Foundation (to S. Nagamatsu).

\section{References}

1. Rothman JE (1994) Mechanisms of intracellular protein transport. Nature 372:55-63

2. Sudhof TC (1995) The synaptic vesicle cycle: a cascade of protein-protein interactions. Nature 375:645-653

3. Jacobsson G, Bean AJ, Scheller RH et al. (1994) Identification of synaptic proteins and their isoform mRNAs in compartments of pancreatic endocrine cells. Proc Natl Acad Sci USA 91:12487-12491

4. Wheeler MB, Sheu L, Ghai M et al. (1996) Characterization of SNARE protein expression in $\beta$ cell lines and pancreatic islets. Endocrinology 137:1340-1348

5. Nagamatsu S, Fujiwara T, Nakamichi Y et al. (1996) Expression and functional role of syntaxin 1/HPC-1 in pancreatic $\beta$ cells. J Biol Chem 271:1160-1165

6. Sadoul K, Lang J, Montecucco C et al. (1995) SNAP-25 is expressed in islets of Langerhans and is involved in insulin release. J Cell Biol 128:1019-1028

7. Martin F, Moya F, Gutierrez LM, Reig JA, Soria B (1995) Role of syntaxin in mouse pancreatic beta cells. Diabetologia 38:860-863

8. Kiraly-Borri CE, Morgan A, Burgoyne RD, Weller U, Wollheim CB, Lang J (1996) Soluble N-ethymaleimidesensitive-factor attachment protein and N-ethylmaleimideinsensitive factors are required for $\mathrm{Ca}^{2+}$-stimulated exocytosis of insulin. Biochem J 314:199-203

9. Nagamatsu S, Watanabe T, Nakamichi Y, Yamamura C, Tsuzuki K, Matsushima S (1999) $\alpha$-Soluble N-ethylmaleimide-sensitive factor attachment protein is expressed in pancreatic $\beta$ cells and functions in insulin but not $\gamma$-aminobutyric acid secretion. J Biol Chem 274:8053-8060

10. Nagamatsu S, Nakamichi Y, Yamamura C et al. (1999) Decreased expression of t-SNARE, syntaxin 1, and SNAP-25 in pancreatic beta-cells is involved in impaired insulin secretion from diabetic GK rat islets: restoration of decreased t-SNARE proteins improves impaired insulin secretion. Diabetes 48:2367-2373

11. Gaisano HY, Ostenson CG, Sheu L, Wheeler MB, Efendic S (2002) Abnormal expression of pancreatic islet exocytotic soluble $\mathrm{N}$-ethylmaleimide-sensitive factor attachment protein receptors in Goto-Kakizaki rats is partially restored by phlorizin treatment and accentuated by high glucose treatment. Endocrinology 143:4218-4226

12. Zhang W, Khan A, Ostenson CG, Berggren PO, Efendic S, Meister B (2002) Down-regulated expression of exocytotic proteins in pancreatic islets of diabetic GK rats. Biochem Biophys Res Commun 291:1038-1044

13. Lang T, Wacker I, Steyer J et al. (1997) $\mathrm{Ca}^{2+}$-triggered peptide secretion in single cells imaged with green fluorescent protein and evanescent wave microscopy. Neuron 18:857863

14. Rohrbach A (2000) Observing secretory granules with a multiangle evanescent wave microscopy. Biophys J 78: 2641-2654

15. Johns LM, Levitan ES, Shelden EA, Holz RW, Axelrod DJ (2001) Restriction of secretory granule motion near the plasma membrane of chromaffin cells. J Cell Biol 183: 177-190

16. Axelrod D (2001) Total internal reflection fluorescent microscopy in cell biology. Traffic 2:764-774

17. Schmoranzer J, Goulain M, Axelrod D, Simon SM (2000) Imaging constitutive exocytosis with total internal reflection fluorescence microscopy. J Cell Biol 149:23-31

18. Ohara-Imaizumi M, Nakamichi Y, Tanaka T, Ishida H, Nagamatsu S (2002) Imaging exocytosis of single insulin secretory granules with evanescent wave microscopy. J Biol Chem 277:3805-3808

19. Tokunaga M, Kitamura K, Saito K, Iwane AH, Yanagida T (1997) Single molecule imaging of fluorophores and enzymatic reactions achieved by objective-type total internal reflection fluorescence microscopy. Biochem Biophys Res Commun 235:47-53

20. Sako Y, Uyemura T (2002) Total internal reflection fluorescence microscopy for single-molecule imaging in living cells. Cell Struct Funct 27:357-365

21. Ohara-Imaizumi M, Nishiwaki C, Kikuta T, Nagai S, Nakamichi Y, Nagamatsu S (2004) TIRF imaging of docking and fusion of single insulin granule motion in primary pancreatic $\beta$-cells: different behaviour of granule motion between normal and Goto-Kakizaki diabetic rat $\beta$-cells. Biochem J 381:13-18

22. Lang T, Bruns D, Wenzel D et al. (2001) SNAREs are concentrated in cholesterol-dependent clusters that define docking and fusion sites for exocytosis. EMBO J 20: 2202-2213

23. Ohara-Imaizumi M, Nishiwaki C, Kikuta T, Kumakura K, Nakamichi Y, Nagamatsu S (2004) Site of docking and fusion of insulin secretory granules in live MIN6 cells analyzed by TAT-conjugated anti-syntaxin 1 antibody and total internal reflection fluorescence microscopy. J Biol Chem 279:8403-8408

24. Ashery U, Varoqueaux F, Voets T et al. (2000) Munc 13-1 acts as a priming factor for large dense-core vesicles in bovine chromaffin cells. EMBO J 19:3586-3596

25. Rizzoli SO, Betz WI (2004) The structural organization of the readily releasable pool of synaptic vesicles. Science 303:2037-2039

26. Zenisek D, Steyer JA, Almers W (2000) Transport, capture and exocytosis of single synaptic vesicles at active zones. Nature 406:849-854

27. Olofsson CS, Gopel SO, Barg S et al. (2002) Fast insulin secretion reflects exocytosis of docked granules in mouse pancreatic $\beta$-cells. Eur J Physiol 444:43-51

28. Washbourne P, Thompson PM, Carta M et al. (2002) Genetic ablation of the t-SNARE SNAP-25 distinguishes mechanisms of neuroexocytosis. Nat Neurosci 5:19-26

29. Sorensen JB, Nagy G, Varoqueaux F et al. (2003) Differential control of the releasable vesicle pools by SNAP-25 splice variants and SNAP-23. Cell 114:75-86

30. Garvey WT, Olefsky JM, Griffin J, Hamman RF, Kolterman OG (1985) The effect of insulin treatment on insulin secretion and insulin action in type II diabetes mellitus. Diabetes 34:222-234

31. Dean PM (1973) Ultrastructural morphometry of the pancreatic $\beta$-cell. Diabetologia 9:115-119

32. Rorsman P, Renstrom E (2003) Insulin granule dynamics in pancreatic beta cells. Diabetologia 46:1029-1045

33. Braun M, Wendt A, Birnir B et al. (2004) Regulated exocytosis of GABA-containing synaptic-like microvesicles in pancreatic $\beta$-cells. J Gen Physiol 123:191-204 